DTP-92/11

February, 1992

\title{
UNIVERSAL FIELD EQUATIONS WITH REPARAMETRISATION INVARIANCE
}

\author{
D.B. FAIRLIE and J. GOVAERTS \\ Department of Mathematical Sciences \\ University of Durham, Durham DH1 3LE, England
}

\begin{abstract}
New reparametrisation invariant field equations are constructed which describe $d$ brane models in a space of $d+1$ dimensions. These equations, like the recently discovered scalar field equations in $d+1$ dimensions, are universal, in the sense that they can be derived from an infinity of inequivalent Lagrangians, but are nonetheless Lorentz (Euclidean) invariant. Moreover, they admit a hierarchical structure, in which they can be derived by a sequence of iterations from an arbitrary reparametrisation covariant Lagrangian, homogeneous of weight one. None of the equations of motion which appear in the hierarchy of iterations have derivatives of the fields higher than the second.

The new sequence of Universal equations is related to the previous one by an inverse function transformation. The particular case of $d=2$, giving a new reparametrisation invariant string equation in 3 dimensions is solved.
\end{abstract}




\section{Introduction}

In a recent paper with $\mathrm{A}$. Morozov ${ }^{[1]}$, we discovered an equation for a single field $\phi$ in $d$ dimensions with some remarkable properties.

1) It is invariant under field redefinitions.

2) It can be derived from an infinity of inequivalent Lagrangians (hence is Universal).

3) It involves no derivatives higher than the second.

4) It is the penultimate step in an iterative hierarchy of equations, starting from any Lagrangian, dependent only on first derivatives of the field and homogeneous of weight one.

We have now found an equation for $d+1$ fields dependent on $d$ variables which satisfy $2,3,4$ above. The requirement 1 is replaced by reparametrisation invariance in the $d$ base space variables. Furthermore there is a transformation which takes one equation into the other. The cases $\mathrm{d}=2,3$ (a new string in 3 dimensions, a new membrane in 4 ) have some physical importance, and may cast further light upon problems of quantum gravity, and perhaps also classical gravity, as the equations are related to the Plebanski equation ${ }^{[2]}$.

The original Universal Field Equation in [1] takes the form

$$
\operatorname{det}\left(\begin{array}{ccccc}
0 & \phi_{1} & \phi_{2} & \ldots & \phi_{d} \\
\phi_{1} & \phi_{11} & \phi_{12} & \ldots & \phi_{1 d} \\
\phi_{2} & \phi_{12} & \phi_{22} & \ldots & \phi_{2 d} \\
. & . & . & \ddots & . \\
\dot{\phi}_{d} & \phi_{1 d} & \phi_{2 d} & \ldots & \phi_{d d}
\end{array}\right)=0
$$

or, in short

$$
\operatorname{det}\left(\begin{array}{cc}
0 & \phi_{k} \\
\phi_{l} & \phi_{k l}
\end{array}\right)
$$

Here $\phi_{k}, \phi_{k l}$ denote partial derivatives $\frac{\partial \phi}{\partial x_{k}}, \frac{\partial^{2} \phi}{\partial x_{k} \partial x_{l}}$ respectively.

It is shown in [1] that equation (1.1) follows as the end result of the following iterative construction of a hierarchy of equations, starting from an arbitrary generic Lagrangian.

Suppose $\mathcal{L}_{0}\left(\phi_{j}\right), j=1, \ldots d$ is a Lagrangian which depends only upon first derivatives

of the field $\phi$, and is homogeneous of weight one, and the matrix $M_{i j}=\frac{\partial^{2} \mathcal{L}_{0}\left(\phi_{k}\right)}{\partial \phi_{i} \partial \phi_{j}}$ is of maximal rank $d-1$, which is the generic case. Denote by $\mathcal{E}$ the Euler differential operator

$$
\mathcal{E}=-\frac{\partial}{\partial \phi}+\partial_{i} \frac{\partial}{\partial \phi_{i}}-\partial_{i} \partial_{j} \frac{\partial}{\partial \phi_{i j}} \ldots
$$

(In principle the expansion continues indefinitely but it is sufficient for our purposes to terminate at the stage of second derivatives $\phi_{i j}$ ).

Now consider the sequence of equations of motion;

$$
\begin{aligned}
\mathcal{E} \mathcal{L}_{0} & =0 \\
\mathcal{E} \mathcal{L}_{0} \mathcal{E} \mathcal{L}_{0} & =0 \\
\mathcal{E} \mathcal{L}_{0} \mathcal{E} \mathcal{L}_{0} \mathcal{E} \mathcal{L}_{0}=0 & \text { etc. }
\end{aligned}
$$


Then this sequence terminates after $d$ iterations when the left hand side vanishes identically. At the penultimate step the resulting equation of motion is universal; i.e. is independent of the details of $\mathcal{L}_{0}$, and is in fact proportional to the equation (1.1). Among the remarkable features of this construction are the consequences that the equations involve nothing further than second derivatives of the field $\phi$ and although the starting Lagrangian need have no symmetry, the final equation is $G L(d)$ invariant. If the homogeneity requirement is dropped, then the sequence results after $d$ iterations with the equation, independently of the initial Lagrangian,

$$
\operatorname{det} \frac{\partial^{2} \phi}{\partial x_{k} \partial x_{l}}=0
$$

and terminates at the next stage in the iteration.

The very nature of our construction implies the existence of an infinite number of conserved quantities for our Universal Equation which take the form of inequivalent Lagrangians, (i.e. Lagrangians which do not differ by divergences). Recently we succeeded in extending these results in the following manner; we discovered a Universal Equation for $d+1$ fields in $d$ dimensions with the property of covariance up to an overall factor under reparametrisation of the base space. That there is only one such equation rather than $d+1$ is a consequence of Ward identities for reparametrisation invariance.

\section{Reparametisation Invariance and Ward identities}

Consider a Lagrangian density $\mathcal{L}\left(\phi_{i}^{a}, \phi_{i j}^{a}\right)$ for $\mathcal{D}$ fields dependent on $d$ co-ordinates such that

$$
\mathcal{L}\left(R_{i j} \phi_{j}^{a}, R_{i k} R_{j l} \phi_{k l}^{a}+T_{i j}^{k} \phi_{k}^{a}\right)=\left(\operatorname{det} R_{i j}\right)^{\alpha} \mathcal{L}\left(\phi_{i}^{a}, \phi_{i j}^{a}\right)
$$

given any coefficients $R_{i j}, T_{i j}^{k}=T_{j i}^{k}$. In consequence of differentiation with respect to those parameters we have the identities

$$
\begin{aligned}
\phi_{j}^{a} \frac{\partial \mathcal{L}}{\partial \phi_{i}^{a}}\left(\phi_{k}^{b}, \phi_{k l}^{b}\right)+2 \phi_{j k}^{a} \frac{\partial \mathcal{L}}{\partial \phi_{i k}^{a}}\left(\phi_{k}^{b}, \phi_{k l}^{b}\right) & =\alpha \delta_{i j} \mathcal{L}\left(\phi_{i}^{a}, \phi_{i j}^{a}\right) \\
\phi_{k}^{a} \frac{\partial \mathcal{L}}{\partial \phi_{i j}^{a}}\left(\phi_{k}^{b}, \phi_{k l}^{b}\right) & =0 .
\end{aligned}
$$

Under a general reparametrisation of co-ordinates of the form

$$
x_{i} \rightarrow y_{i}=y_{i}\left(x_{j}\right), \phi^{a}\left(x_{i}\right) \rightarrow \tilde{\phi}^{a}\left(y_{i}\right)=\phi^{a}\left(x_{i}\right)
$$

we have

$$
\begin{aligned}
\tilde{\phi}_{i}^{a}=R_{i j} \phi_{j}^{a}, \quad \tilde{\phi}_{i j}^{a}=R_{i k} R_{j l} \phi_{k l}^{a}+T_{i j}^{k} \phi_{k}^{a} \\
\text { with } \quad R_{i j}=\frac{\partial x_{j}}{\partial y_{i}}, \quad T_{i j}^{k}=\frac{\partial^{2} x_{k}}{\partial y_{i} \partial y_{j}} .
\end{aligned}
$$

The case $\alpha=1$ corresponds to a reparametrisation invariant action. 
Given a Lagrangian density with the above properties, consider the action of the Euler derivative;

$$
\mathcal{E}_{a} \mathcal{L}=\partial_{i} \frac{\partial \mathcal{L}}{\partial \phi_{i}^{a}}-\partial_{i} \partial_{j} \frac{\partial \mathcal{L}}{\partial \phi_{i j}^{a}} .
$$

We then have

$$
\begin{aligned}
\phi_{i}^{a} \mathcal{E}_{a} \mathcal{L}= & \phi_{i}^{a} \partial_{j}\left\{\frac{\partial \mathcal{L}}{\partial \phi_{j}^{a}}-\partial_{k} \frac{\partial \mathcal{L}}{\partial \phi_{j k}^{a}}\right\} \\
= & \partial_{j}\left\{\phi_{i}^{a} \frac{\partial \mathcal{L}}{\partial \phi_{j}^{a}}+\phi_{i k}^{a} \frac{\partial \mathcal{L}}{\partial \phi_{j k}^{a}}-\partial_{k}\left[\phi_{i}^{a} \frac{\partial \mathcal{L}}{\partial \phi_{j k}^{a}}\right]\right\} \\
& -\phi_{i j}^{a}\left\{\frac{\partial \mathcal{L}}{\partial \phi_{j}^{a}}-\partial_{k} \frac{\partial \mathcal{L}}{\partial \phi_{j k}^{a}}\right\} \\
= & \partial_{j}\left\{\phi_{i}^{a} \frac{\partial \mathcal{L}}{\partial \phi_{j}^{a}}+2 \phi_{i k}^{a} \frac{\partial \mathcal{L}}{\partial \phi_{j k}^{a}}\right\}-\partial_{i} \mathcal{L}-\partial_{j} \partial_{k}\left[\phi_{i}^{a} \frac{\partial \mathcal{L}}{\partial \phi_{j k}^{a}}\right],
\end{aligned}
$$

which, by using the identities $(2.2 \mathrm{a}, 2.2 \mathrm{~b})$ reduces to

$$
\phi_{i}^{a} \mathcal{E}_{a} \mathcal{L}=(\alpha-1) \partial_{i} \mathcal{L} .
$$

Whenever $\alpha=1$, corresponding to a reparametriation invariant action, we have the associated $d$ Ward identities:

$$
\phi_{i}^{a} \mathcal{E}_{a} \mathcal{L}=0 .
$$

Consequently there are only $\mathcal{D}-d$ independent equations of motion.

\section{The Case $\mathcal{D}=d+1$}

The situation $\mathcal{D}=d+1$ includes that of a string in 3 dimensions, or a membrane in 4. On account of reparametrisation invariance there is only one independent equation of motion. It is convenient to define the Jacobians

$$
J_{a}=(-1)^{d} \epsilon_{a b_{1} b_{2} \ldots b_{d}} \phi_{1}^{b_{1}} \phi_{2}^{b_{2}} \ldots \phi_{d}^{b_{d}} .
$$

The following properties hold;

$$
\begin{aligned}
& \text { i) } \sum_{a} \phi_{i}^{a} J_{a}=0 \quad \forall i, \\
& \text { ii) } \quad \sum_{a} \phi_{i}^{a} \frac{\partial J_{a}}{\partial \phi_{j}^{b}}+\delta_{i j} J_{b}=0, \\
& \text { iii) } \frac{\partial J_{a}}{\partial \phi_{i}^{b}}=-\frac{\partial J_{b}}{\partial \phi_{i}^{a}} .
\end{aligned}
$$

The identities $\phi_{k}^{a} \frac{\partial \mathcal{L}}{\partial \phi_{i j}^{a}}\left(\phi_{i}^{b}, \phi_{i j}^{b}\right)=0$ show that in the present case we must have (no summation over $a$ )

$$
\frac{\partial \mathcal{L}}{\partial \phi_{i j}^{a}}\left(\phi_{k}^{b}, \phi_{k l}^{b}\right)=J_{a} K_{i j}^{a}\left(\phi_{i}^{a}, \phi_{i j}^{a}\right) .
$$


This result suggests that reparametrisation invariant actions which depend upon $\phi_{i j}^{a}$ must involve those variables in the combination $\Phi_{i j}=\sum_{a} \phi_{i j}^{a} J_{a}$. Indeed it turns out that the Universal field equation in this case is simply

$$
\operatorname{det}\left(\Phi_{i j}\right)=0
$$

\section{A hierarchy for $\mathcal{D}=d+1$}

Consider a Lagrangian density $\mathcal{L}_{0}\left(J_{a}\right)$ such that

$$
\mathcal{L}_{0}\left(\lambda J_{a}\right)=\lambda \mathcal{L}_{0}\left(J_{a}\right)
$$

The corresponding action is then reparametrisation invariant. We also have;

$$
\begin{aligned}
\frac{\partial \mathcal{L}_{0}}{\partial J_{b}}\left(J_{a}\right) J_{b} & =\mathcal{L}_{0}\left(J_{a}\right), \\
\frac{\partial^{2} \mathcal{L}_{0}}{\partial J_{a} \partial J_{b}}\left(J_{c}\right) J_{b} & =0, \\
\frac{\partial^{2} \mathcal{L}_{0}}{\partial J_{a} \partial J_{b}}\left(J_{c}\right) & =C_{i j}\left(\phi_{k}^{c}\right) \phi_{i}^{a} \phi_{j}^{a},
\end{aligned}
$$

where $C_{i j}\left(\phi_{k}^{c}\right)=C_{j i}\left(\phi_{k}^{c}\right)$ and is given by

$$
C_{i j}=\frac{1}{2 J_{a} J_{b}}\left[\frac{\partial^{2} \mathcal{L}_{0}}{\partial \phi_{i}^{a} \partial \phi_{j}^{b}}+\frac{\partial^{2} \mathcal{L}_{0}}{\partial \phi_{j}^{a} \partial \phi_{i}^{b}}\right], \quad \forall a, b, i, j .
$$

The equations of motion are

$$
\mathcal{E}_{a} \mathcal{L}_{0}=\frac{\partial^{2} \mathcal{L}_{0}}{\partial J_{b} \partial J_{c}} \frac{\partial J_{b}}{\partial \phi_{i}^{a}} \frac{\partial J_{c}}{\partial \phi_{j}^{d}} \phi_{i j}^{d}=J_{a} C_{i j} \Phi_{i j} .
$$

This shows that the independent equation of motion is always given by

$$
\frac{1}{J_{a}} \mathcal{E}_{a} \mathcal{L}_{0}=C_{i j} \Phi_{i j}, \quad \forall a
$$

This suggests that, in analogy to the case $\mathcal{D}=1, d$ arbitrary, we consider the generating function $\exp \left(\lambda \mathcal{L}_{0} \frac{1}{J_{a}} \mathcal{E}_{a}\right) \cdot \mathcal{L}_{0}$ for any $a$ which generates term by term an iterative sequence of equations of motion. This sequence possesses the following remarkable properties:

1) It is a finite polynomial of order $d$ in $\lambda$.

2) It depends only upon $\phi_{i}^{a}, \phi_{i j}^{a}$, and no higher derivatives.

3) Every term in the polynomial is a Lagrangian density for a reparametrisation invariant action, which is multilinear in $\phi_{i j}^{a}$. 
4) Up to a factor dependent on $\mathcal{L}_{0}$, the coefficient of $\lambda^{d}$ is independent of the details of $\mathcal{L}_{0}$ and hence is Universal. This is the equation (3.4).

This means that the Universal equation may be derived from an infinite number of inequivalent Lagrangians, hence the terminology, and may therefore be integrable. The proof of these statements will be merely sketched here. It depends upon the fact that it turns out that this is not an independent hierarchy of iterative equations but is a transformation of the original case $\mathcal{D}=1, d$ arbitrary. Introduce a new variable $y$ which is in the nature of a co-ordinate, and consider $\mathcal{L}_{0}\left(J_{a}\right)$ where now $\phi^{a}\left(x_{i}, y\right)$ depends upon the auxiliary co-ordinate, but $J_{a}$ is still given by equation (3.1), i.e. it is the Jacobian of all the $\phi^{b}$ excluding $\phi^{a}$ with respect to all the coordinates $x_{i}$, excluding $y$. Then the equations of motion do not involve derivatives with respect to $y$ as $\mathcal{L}_{0}$ depends only upon $\frac{\partial \phi^{a}}{\partial x_{i}}$. Indeed

$$
\left[\partial_{x_{i}} \frac{\partial}{\partial \phi_{i}^{a}}+\partial_{y} \frac{\partial}{\partial \phi_{y}^{a}}\right] \mathcal{L}_{0}=\partial_{x_{i}} \frac{\partial}{\partial \phi_{i}^{a}} \mathcal{L}_{0}\left(J_{a}\right)=\mathcal{E}_{a} \mathcal{L}_{0}\left(J_{a}\right)
$$

Thus if $\mathcal{L}_{0}\left(J_{a}\right)$ is homogeneous of weight one, i.e. it satisfies (4.1), then the action is reparametrisation invariant in the $x$ 's and takes the form

$$
\mathcal{S}=\int \prod_{i} d x_{i} d y \mathcal{L}_{0}\left(J_{a}\right)
$$

Now perform the inverse function transformation; i.e. instead of treating $\phi^{a}$ as dependent functions and $x_{i}, y$ as independent, think of $x_{i}, y$ as described as functions of $\phi^{a}$. We have then

$$
\mathcal{S}=\int \prod_{i} d x_{i} d y \mathcal{L}_{0}\left(J_{a}\right) \equiv \int \prod_{a} d \phi^{a} \mathcal{L}_{0}\left(\frac{\partial y}{\partial \phi^{a}}\right) .
$$

Remarkably, on account of the homogeneity properties, all other jacobians cancel in the above transformation of variables. But the transformed action is just of the form of the starting action for the hierarchy discovered in [1]. A Lagrangian $\mathcal{L}_{0}\left(\frac{\partial y}{\partial \phi^{a}}\right)$ of weight one dependent upon only first derivatives has an equation of motion covariant under field redefinitions of $y$. It can be taken as the beginning of an iterative sequence (1.4), which culminates in the Universal field equation (1.1). Clearly it is possible to map each member of the iterative sequence (1.4) into a new iterative sequence, which this time, instead of invariance under field redefinitions, implements reparametrisation invariance for each sequential action. The remarkable feature of this procedure is that all derivatives with respect to $y$ disappear from the transformation of the generating function

$$
\begin{aligned}
{\left[\exp \lambda \mathcal{L}_{0}\left(\frac{\partial y}{\partial \phi^{a}}\right) \mathcal{E}_{y}\right] \mathcal{L}_{0}\left(\frac{\partial y}{\partial \phi^{a}}\right) } & =\mathcal{L}_{0}\left(\frac{\partial y}{\partial \phi^{a}}\right) \operatorname{det}\left[\delta_{a b}+\lambda \frac{\partial^{2} \mathcal{L}_{0}}{\partial X_{a} \partial X_{c}} \frac{\partial^{2} y}{\partial \phi^{c} \partial \phi^{b}}\right] \\
\text { where } \quad X_{a} & =\frac{\partial y}{\partial \phi^{a}} .
\end{aligned}
$$

Suppose this equation is re-expressed in terms of the inverse functions $\phi^{a}\left(x_{i}, y\right)$. Then this results in the following transformed equations $\forall a$;

$$
\exp \left[\lambda \frac{\mathcal{L}_{0}\left(J_{a}\right)}{J_{a}} \mathcal{E}_{a}\right] \mathcal{L}_{0}\left(J_{a}\right)=\mathcal{L}_{0}\left(J_{a}\right) \operatorname{det}\left[\delta_{i j}+\lambda C_{i k}\left(\phi_{i}^{a}\right) \Phi_{k j}\right]=\mathcal{L}\left(\phi_{i}^{a}, \phi_{i j}^{a}, \lambda\right)
$$


where $\mathcal{L}_{0}\left(\lambda J_{a}\right)=\lambda \mathcal{L}_{0}\left(J_{a}\right)$ and $C_{i k}\left(\phi_{i}^{a}\right)$ and $\Phi_{k j}$ are defined by equations (4.3) and $\Phi_{i j}=$ $\sum_{a} \phi_{i j}^{a} J_{a}$. The remarkable feature of this transformation is that all possible dependence of (4.10) on $\phi_{y}^{a}, \phi_{i y}^{a}, \phi_{y y}^{a}$ has disappeared, so $y$ is effectively a parameter as far as equation (4.10) is concerned. The equation

$$
\mathcal{E}_{a} \mathcal{L}\left(\phi_{i}^{a}, \phi_{i j}^{a}, \lambda\right)=\frac{J_{a}}{\mathcal{L}_{0}} \frac{\partial}{\partial \lambda} \mathcal{L}\left(\phi_{i}^{a}, \phi_{i j}^{a}, \lambda\right)
$$

is the generating function for the reparametrisation invariant hierarchy which terminates at the $d$ th level, with the term in $\lambda^{d}$ being

$$
\mathcal{L}_{0}\left(J_{a}\right) \operatorname{det}\left[C_{i k} \Phi_{k j}\right]=\mathcal{L}_{0}\left(J_{a}\right) \operatorname{det}\left[C_{i k}\right] \operatorname{det}\left[\Phi_{k j}\right] .
$$

Since for generic $\mathcal{L}_{0}\left(J_{a}\right), \operatorname{det}\left[C_{i k}\right] \neq 0$, we have the Universal equation of motion

$$
\operatorname{det}\left[\Phi_{i j}\right]=\operatorname{det}\left[\phi_{i j}^{a} J_{a}\right]=0 .
$$

\section{Solutions for $d=2$, Duality}

This is the case of the alternative reparametrisation invariant string in 3 dimensions. It is apparently different from that proposed by Grigore ${ }^{[3]}$ since he does not consider Lagrangians dependent on second derivatives. From the analysis presented in the above section the solutions of the equation $\operatorname{det}\left[\Phi_{i j}\right]=0$ are equivalent to solving the Universal field equation for one variable $y=f$ in three dimensions $X, Y, Z{ }^{[1]}$; viz:

$$
\operatorname{det}\left(\begin{array}{cccc}
0 & f_{X} & f_{Y} & f_{Z} \\
f_{X} & f_{X X} & f_{X Y} & f_{X Z} \\
f_{Y} & f_{X Y} & f_{Y Y} & f_{Y Z} \\
f_{Z} & f_{X Z} & f_{Y Z} & f_{Z Z}
\end{array}\right)=0 .
$$

Solutions of this equation correspond to developable surfaces ${ }^{[4]}$. Such surfaces, with the exception of the plane, cone and cylinder are described by the tangent surface of a curve, i.e. the locus of points on the tangents to an arbitrary curve. It is shown in [4] that such surfaces are two sheeted, with a cusp at the curve in question. Another parametrisation of such a surface is given by $X=X(\sigma, \tau), Y=Y(\sigma, \tau), Z=Z(\sigma, \tau)$, the string formulation, which satisfies an equation of the form (4.13). The cone and cylinder may be taken to represent closed string solutions, in which a closed string propagates with and without expansion. These two alternative descriptions of the string, in a non parametric form as a surface, and the parametrised form are reminiscent of the attempt of Morris ${ }^{[5]}$ to develop ordinary string theory in terms of intersections of surfaces. In this second form the equation is related to Plebanski's equation for self-dual Riemannian metrics ${ }^{[2]}$.

Finally we should mention the second case in [1] which we found which admits a formulation in terms of an infinite number of inequivalent Lagrangians. It is the case of 
$\mathcal{D}=d$ fields in $d+1$ variables. There are $d$ equations of motion, invariant under field redefinitions of the form

$$
\epsilon_{i_{1} i_{2} \ldots i_{d+1}} \epsilon_{j_{1} j_{2} \ldots j_{d+1}} \prod_{r=1}^{d} \phi_{i_{r}}^{b_{r}} \prod_{s=1}^{d} \phi_{j_{s}}^{c_{s}} \phi_{i_{d+1} j_{d+1}}^{a} .
$$

Under the transformation discussed previously, these equations go over into the $d$ independent equations

$$
\frac{\partial^{2} x_{i}}{\partial y^{2}} \frac{\partial x_{j}}{\partial y}=\frac{\partial^{2} x_{j}}{\partial y^{2}} \frac{\partial x_{i}}{\partial y}, \quad \forall i, j
$$

These equations are covariant under reparametrisations in the variable $y$. They may be regarded as a dual version of the $\mathcal{D}=d+1, d=d$ theory presented here.

\section{Conclusions.}

This paper and its predecessor ${ }^{[1]}$ have revealed the existence of field theory models which possess many interlinked and unexpected properties; reparametrisation invariance, infinitely many conservation laws, absence of higher derivatives, and the hierarchy property. It is not at all clear at this stage which is fundamental, and which are inevitable consequences. Certainly the fact that these equations may be derived from an infinity of Lagrangians, inequivalent in so far as they are not related by divergences makes them interesting objects of study in their relation to topological field theories ${ }^{[6]}$ and variational procedures $^{[7]}$. It is also not clear at the moment whether there is any connection with the geometrical realisations of $W$ algebras ${ }^{[8]}$, or indeed gravity theories ${ }^{[9]}$.

The extension of those ideas to arbitrary choices of target space and base space dimension remains elusive. It is relatively easy to write equations with the correct covariance or reparametrisation properties, but there is a superfluity of such equations (3 instead of 2 for $\mathcal{D}=4, d=2$ ), and there is no sign of the hierarchy property. Nonetheless the solution space is non empty. The success of the $\mathcal{D}=3, d=2$ model may owe its existence to the invariant

$$
\epsilon_{a b c} \epsilon_{i k} \phi_{i}^{a} \phi_{j}^{b} \phi_{k j}^{c}
$$

We hope to return to those questions and give a more detailed account of the results of the present paper in the near future.

\section{Acknowledgement}

This research was supported by the S.E.R.C. award of a Research Assistantship to J.Govaerts. 


\section{REFERENCES}

[1] Fairlie D. B., Govaerts J. and Morozov A., Universal Field Equations with Covariant Solutions, Durham preprint DTP-91/55, hepth-9110022 (October 1991), to appear in Nuclear Physics B.

[2] Finley J. D. and Plebanski J. F., Jour. Math. Phys. 17 (1976) 585.

[3] Grigore D. R., A Derivation of the Nambu-Goto Action from Invariance Principles, preprint CERN-TH.6101/91 (May 1991).

[4] Eisenhart L. P., An Introduction to Differential Geometry, Princeton University Press (1940) 59.

[5] Morris T. W., Phys. Lett. B 202 (1988) 222; Nucl. Phys. B 331 (1990) 694.

[6] Witten E., Comm. Math. Phys. 117 (1988) 353; ibid. 118 (1988) 411.

[7] Olver E., Applications of Lie Groups to Differential Equations Graduate Texts in Mathematics, 107, Springer Verlag (1986), p. 252.

[8] Gervais J.-L. and Matsuo Y., preprint LPTENS-91/35, NBI-HE-91/50, hepth-9201026 (January 1992).

[9] Hull C. M., The Geometry of $W$-Gravity, preprint QMW/PH/91/6 (June 1991). 